Check for updates

AUTHORS:

Nonkululeko Mfeka' (iD

Reckson A. Mulidzi ${ }^{2}$ iD

Francis B. Lewu ${ }^{1}$ iD

\section{AFFILIATIONS:}

'Department of Agriculture, Cape Peninsula University of Technology, Cape Town, South Africa

${ }^{2}$ Soil and Water Science, Agricultura Research Council, Stellenbosch, South Africa

\section{CORRESPONDENCE TO:}

Nonkululeko Mfeka

EMAIL:

mfekan@cput.ac.za

\section{DATES:}

Received: 16 Feb. 2018

Revised: 08 June 2018

Accepted: 23 Aug. 2018

Published: 30 Jan. 2019

\section{HOW TO CITE:}

Mfeka N, Mulidzi RA, Lewu FB.

Growth and yield parameters of three cowpea (Vigna unguiculata L. Walp) lines as affected by planting date and zinc application rate. S Afr J Sci. 2019:115(1/2), Art. \#4474, 8 pages. https://doi.org/10.17159/ sajs.2019/4474

\section{ARTICLE INCLUDES: \\ 囚 Peer review}

$\square$ Supplementary material

\section{DATA AVAILABILITY:}

冈 Open data set

$\square$ All data included

$\square$ On request from authors

$\square$ Not available

$\square$ Not applicable

\section{EDITOR:}

Teresa Coutinho (ID)

\section{KEYWORDS:}

vegetative parameters; crop production; zinc fertiliser; Western Cape

FUNDING:

Cape Peninsula University of Technology; Agricultural Research Council (South Africa); National Research Foundation (South Africa)

(c) 2019. The Author(s). Published under a Creative Commons Attribution Licence.

\title{
Growth and yield parameters of three cowpea (Vigna unguiculata L. Walp) lines as affected by planting date and zinc application rate
}

\begin{abstract}
Cowpea is one of the most important food legumes in most African countries. Cowpea is a valuable source of dietary protein for both humans and their livestock. There is limited information available on cowpea production and suitable agronomic practices, such as planting date, to best suit different environmental conditions in South Africa. Therefore, the objective of this study was to evaluate the effect of two locations on cowpea production and the effect of planting date as affected by zinc application rate. Field experiments were conducted at two locations (Bien Donne' and Nietvoorbij) in the Western Cape Province of South Africa, using two planting dates (2 October and 2 November), three cowpea lines (Veg1, M217 and Qukawa) and three zinc (Zn) fertiliser application levels $(0 \mathrm{~kg} / \mathrm{ha}, 15 \mathrm{~kg} / \mathrm{ha}$ and $30 \mathrm{~kg} / \mathrm{ha}$ ) during the 2015 growing season. The experimental design was a randomised complete block with five replicates. The results showed that Veg1 and Qukawa lines performed significantly better in both vegetative and reproductive parameters when compared to M217 at both locations. Application of zinc fertiliser significantly $(p<0.05)$ affected seed iron content in Veg1 and M217 at Bien Donne' and seed iron content in M217 and zinc content in Veg1 at Nietvoorbij.
\end{abstract}

\section{Significance:}

- Cowpea lines Veg1 and Qukawa were the best performing lines in all parameters measured, making these two lines suitable for dual purpose cultivation.

- Planting cowpea in November, rather than October, increased the crop production efficiency.

- Cowpea showed a better overall total yield in the sandy soil of Bien Donne' than in the sandy loam clay soil of Nietvoorbij.

\section{Introduction}

Cowpea (Vigna unguiculata L. Walp) is a significant grain and fodder pulse grown around the world..$^{1,2}$ It serves as a dual purpose grain legume crop, providing food for human consumption and fodder for livestock. ${ }^{3}$ This crop is successful in most regions because of its ability to survive in low fertile soils ${ }^{4}$, and withstand alkaline soils ${ }^{5}$. Cowpea is a staple crop in most African countries. ${ }^{6}$ According to Gomez ${ }^{7}$, Africa is the leading continent in cowpea production at $68 \%$, followed by Brazil at $17 \%$, Asia at $3 \%$, the USA at $2 \%$, with the remaining $10 \%$ produced by the rest of the world. Africa alone accounts for 10 million hectares under cowpea production ${ }^{8}$ and the crop is indigenous to Africa ${ }^{3,9}$. Cowpea seed as well as the vegetative parts make a major nutritional contribution to the human diet. ${ }^{10}$ The seed contains $25 \%$ protein and $64 \%$ carbohydrates $^{11,12}$, with $27-34 \%$ protein in the leaves ${ }^{13,14}$. In South Africa, cowpea is mainly cultivated in the Limpopo, Mpumalanga, North-West and KwaZulu-Natal Provinces. ${ }^{15}$ Cultivation of cowpea is mainly to maintain the nitrogen status of soil, thereby reducing the costs of commercial nitrogen fertilisers. ${ }^{16}$ According to Bloem et al. ${ }^{17}$, biological nitrogen fixation of leguminous crops is an affordable and sustainable biological method to enhance soil fertility that is used by South African farmers to increase crop yield.

Cowpea has been identified as a neglected and underutilised crop species, with further research required in some parts of Africa where there is limited information on its cultivation agronomic practices and seed handling as it is of relatively less commercial interest. ${ }^{18-20}$ In South Africa, there is very limited information on the cultivation and agronomy of cowpea because of a lack of interest and funding. ${ }^{21}$ Recent results have shown that cowpea is still an underutilised crop. ${ }^{22}$ According to Quass ${ }^{23}$, no coordinating body exists for cowpea production in South Africa and consequently there are no available data on production. This limitation results in poor supply of good quality seed. ${ }^{24}$ To the best of our knowledge, there is no reported information on the cultivation practices of cowpea in the Mediterranean climate of the Western Cape Province of South Africa. In this study, we therefore present the first report on the cultivation of cowpea under two planting dates and at different levels of zinc application in the Cape Winelands region of the Western Cape Province.

The Winelands region contributes to the highest cultivation of grapes which needs high soil fertility. Farmers in the Western Cape Province mostly practice mixed farming which predominantly includes livestock. Integration of cowpea in the vine-based cropping system of the Western Cape can improve nitrogen and carbon supply to the soil with a resultant reduction in the use of chemical fertiliser. The dual purpose cowpea tested in the trial will significantly increase plant-based protein for humans and livestock; and also improve soil fertility for ultimately improved grape production under limited application of mineral fertiliser.

\section{Material and methods}

\section{Cowpea lines and study area}

Three cowpea lines were used in the study: Veg1, M217 and Qukawa. These lines were obtained from the Genebank of the Vegetable and Ornamental Plant Institute of the Agricultural Research Council, Pretoria, South Africa. Morphological traits of the three lines were recorded according to the International Board for Plant Genetic Resources $^{25}$ as presented in Table 1. The field experiment was conducted at the Agricultural Research Council in Stellenbosch, Western Cape. The experiment was done on two Agricultural Research Council research farms -

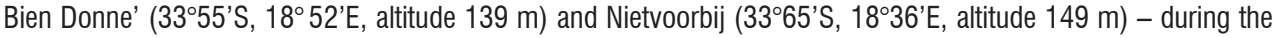


Table 1: $\quad$ Qualitative and quantitative traits of the three cowpea lines

\begin{tabular}{|c|c|c|c|c|c|c|c|c|c|c|c|c|c|c|c|c|}
\hline \multirow{2}{*}{ Line } & & \multicolumn{15}{|c|}{ Trait } \\
\hline & GP & GH & LC & FC & NMB & PPP & PA & PL & PS & NPPP & PC & SPP & SC & ST & SS & sW \\
\hline Veg1 & 2 & 3 & 7 & 4 & 4.3 & 3 & 3 & 15.8 & 3 & 40.7 & 1 & 15.1 & 3 & 1 & 5 & 11.7 \\
\hline M217 & 1 & 2 & 5 & 2 & 4.5 & 3 & 5 & 10.1 & 5 & 26.2 & 2 & 8.4 & 1 & 3 & 1 & 14.3 \\
\hline Qukawa & 2 & 5 & 5 & 4 & 4.4 & 2 & 3 & 17.25 & 3 & 18.7 & 3 & 10.8 & 2 & 1 & 1 & 14.1 \\
\hline
\end{tabular}

GP, growth pattern: 1, determinate, 2, indeterminate; GH, growth habit: 1, acute erect (branches form acute angle with main stem), 2, erect (branching angle less acute than above), 3, semi-erect (branches perpendicular to main stem, but do not touch ground), 4, intermediate (most lower branches touch the ground), 5, semi-prostrate (main stem reaches $20 \mathrm{~cm}$ or more), 6, prostrate (plants flat on ground), 7, climbing; LC, leaf colour: 3, pale green, 5, intermediate green, 7, dark green; FC, flower colour: 1, white, 2, yellow, 3, red, 4, purple; NMB, number of main branches: average of 10 randomly selected plants; $P P P$, pods per peduncle, average of mature pods from 10 randomly selected plants: $1=1-2,2=2-3,3=3-4$; PA, pod attachment: 3, pendant, 5, 30-90 down from erect, 7, erect; $P$, pod length (cm): average of 10 mature pods from 10 randomly selected plants; PS, pod shape: 0 , straight, 3, slightly curved, 5, curved, 7, coiled; NPPP, number of pods per plant: average number of pods from 10 selected plants; PC, pod colour: 1, pale tan or straw, 2, dark tan, 3, dark brown; SPP, seed per pod: average of 10 pods; SC, seed colour: 1, cream, 2, brown, 3, grey; 4, black or dark purple; 5, other; ST, seed texture: 1, smooth, 3, smooth to rough, 5, rough, 7, rough to wrinkled, 9, wrinkled; SS, seed shape: 1, kidney, 2, ovoid, 3, crowder, 4, globose, 5, rhomboid; SW, seed weight (g): weight of 100 seeds per treatment

2015 summer growing season. The soil at Bien Donne' has a sandy loam texture with a relatively high percentage of sand $(77 \%)$ and low clay percentage (14.2\%). The soil at Nietvoorbij is a sandy clay loam with $69.7 \%$ sand and $20.6 \%$ clay. The Western Cape Province has a Mediterranean climate and the study area has an annual rainfall of $278 \mathrm{~mm}$, of which approximately $178 \mathrm{~mm}$ falls from March to August. ${ }^{26}$

\section{Experimental design and treatment}

The trial layout was a randomised complete block design replicated five times with an experimental plot size of $2 \mathrm{~m} \times 1 \mathrm{~m}$. Cowpea seeds were sown at an inter-row spacing of $0.4 \mathrm{~m}$ and intra-row spacing of $0.2 \mathrm{~m}$. The experiment had four factors: the two planting dates $(2$ October and 2 November 2015), two soil types (sandy clay loam and sandy loam soil), three cowpea lines ( Veg1, M217 and Qukawa) and three levels of zinc $(1=0 \%$ or control, $2=50 \%$ and $3=100 \%)$. Soil application of zinc sulfate $\left(\mathrm{ZnSO}_{4}\right)$ was done at flowering. The trial consisted of nine treatments per location per planting date (Table 2).

\section{Cultivation practices and management}

The experimental sites were ploughed, disc-harrowed and levelled into experimental plots. Two seeds were sown per stand using the dibbling method. Plants were later thinned to one plant per stand when the second trifoliate leaves had unfolded. Hand weeding of experimental units was done at 4 weeks after germination and subsequently at 3-week intervals as needed. Cutworm was controlled by applying Cutworm Bait 4 weeks after planting, while aphids were controlled with Kemprin 200 EC sprayed at a rate of $1.0 \mathrm{~mL} / \mathrm{L}$ water using a backpack sprayer.

\section{Data collection and analysis}

Vegetative data were taken from the inner two rows at 2-week intervals. The number of germinated plants was recorded at 7 days after planting and germination (GP) was calculated as a percentage of germinated plants per experimental unit using the formula of Pahla et al. ${ }^{27}$.

$\mathrm{GP}=$ no. of $\mathrm{g} / \mathrm{T} \times 100$,

where $g$ is the number of germinated plants and $T$ is the total number of seeds planted.

The number of leaves borne on each plant at full leaf maturity was counted and the number of branches per plant was obtained by counting the main stem of the sample plants. Plant height $(\mathrm{m})$ was measured from the main stem, from ground level to the tip of the plant using a meter ruler.

Reproductive parameters were collected after harvesting the two middle rows from each experimental unit. Matured pods from sample plants were counted per plant, weighed $(\mathrm{g})$ and measured in metres. Hundredseed weight (g) was determined by randomly counting 100 seeds from threshed pods per experimental unit using a digital weighing scale.
Table 2: Treatment details and codes

\begin{tabular}{c|c|c|c}
\hline \hline Cowpea line & Name & Zinc level & Application rate \\
\hline \hline 1 & Veg1 & 1 & $0 \%$ Control \\
\hline 1 & Veg1 & 2 & $50 \% \mathrm{Zn}$ \\
\hline 2 & Veg1 & 3 & $100 \% \mathrm{Zn}$ \\
\hline 2 & M217 & 1 & $0 \%$ Control \\
\hline 2 & M217 & 2 & $50 \% \mathrm{Zn}$ \\
\hline 3 & M217 & 3 & $100 \% \mathrm{Zn}$ \\
\hline 3 & Qukawa & 1 & $0 \%$ Control \\
\hline 3 & Qukawa & 2 & $50 \% \mathrm{Zn}$ \\
\hline
\end{tabular}

Data on vegetative and reproductive parameters as well as the mineral content of the seed were subjected to an analysis of variance (ANOVA) using SAS. ${ }^{28}$ Treatments were tested at a $5 \%$ level of significance and differences between treatments were separated using least significant difference and Duncan's Multiple Range Test of the SAS 2012 package.

\section{Results}

\section{Vegetative parameters}

Cowpea lines had a significant difference $(p<0.05)$ in germination percentage. Line 1 showed significantly more $(p<0.05)$ germination than Line 2 during the October planting date. Germination percentage was generally higher during the second planting date for both locations (Table 3). Figure 1 shows germination of the three cowpea lines as affected by location.

The number of leaves per plant was not significantly different between cowpea lines and $\mathrm{Zn}$ levels at the two locations. Line 1 at $\mathrm{Zn}$ level 2 produced the most branches per plant at Bien Donne' (Table 4). Lines 1 and 3 produced taller plants than Line 2 at both locations. The second planting date had a significant effect on all the vegetative parameters measured at Nietvoorbij. 
Table 3: $\quad$ Effect of planting date on the germination rate of three cowpea lines at two locations

\begin{tabular}{c|c|c|c|c}
\hline \hline \multirow{2}{*}{ Cowpea line } & \multicolumn{2}{|c|}{ Bien Donne' } & \multicolumn{2}{c}{ Nietvoorbij } \\
\cline { 2 - 5 } & October & November & 0ctober & November \\
\hline \hline 1 & $89^{\mathrm{a}}$ & $94^{\mathrm{a}}$ & $81^{\mathrm{a}}$ & $97^{\mathrm{a}}$ \\
\hline 2 & $75^{\mathrm{b}}$ & $93^{\mathrm{a}}$ & $53^{\mathrm{b}}$ & $93^{\mathrm{a}}$ \\
\hline 3 & $85^{\mathrm{ab}}$ & $93^{\mathrm{a}}$ & $79^{\mathrm{a}}$ & 15.9 \\
\hline$L_{0.05}$ & 12.25 & 15.85 & 15 & 9 \\
\hline
\end{tabular}

$L S D_{0.05}$ least significant difference; L1, Veg1; L2, M217; L3, Qukawa; T1, 0\% Zn; T2, Zn at 50\%; T3, Zn at 100\%.

Means with different superscript letters within the same column are significantly different $(\mathrm{p}<0.05)$.
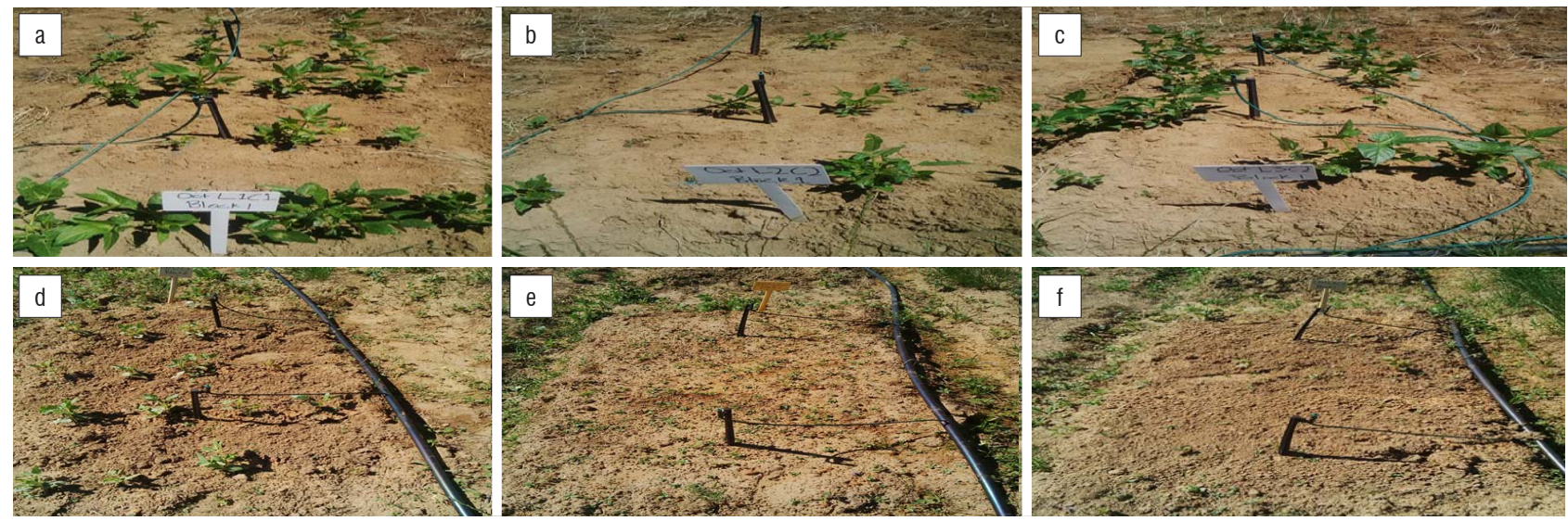

Figure 1: Photograph showing vegetative growth of the three cowpea lines $(a, d)$ Veg1, $(b, e)$ M217 and $(c, f)$ Qukawa at $(a-c)$ Bien Donne' and (d-f) Nietvoorbj at 8 weeks after planting for the first planting date.

Table 4: $\quad$ Effect of cowpea line and zinc application rate on vegetative parameters at two locations

\begin{tabular}{|c|c|c|c|c|c|c|c|}
\hline \multirow[b]{2}{*}{ Cowpea line } & \multirow[b]{2}{*}{ Zinc level } & \multicolumn{3}{|c|}{ Bien Donne' } & \multicolumn{3}{|c|}{ Nietvoorbij } \\
\hline & & Number of leaves & $\begin{array}{l}\text { Number of } \\
\text { branches }\end{array}$ & $\begin{array}{l}\text { Plant height } \\
\text { (cm) }\end{array}$ & Number of leaves & $\begin{array}{l}\text { Number of } \\
\text { branches }\end{array}$ & $\begin{array}{l}\text { Plant height } \\
\text { (cm) }\end{array}$ \\
\hline 1 & 1 & $65.26^{a}$ & $7.06^{\mathrm{a}}$ & $15.52^{\mathrm{a}}$ & $59.75^{a}$ & $5.17^{\mathrm{a}}$ & $15.60^{a}$ \\
\hline 1 & 2 & $67.37^{\mathrm{a}}$ & $7.21^{\mathrm{a}}$ & $14.05^{\mathrm{a}}$ & $63.26^{\mathrm{a}}$ & $4.93^{\mathrm{a}}$ & $14.60^{\mathrm{a}}$ \\
\hline 1 & 3 & $50.06^{a}$ & $6.36^{\mathrm{ab}}$ & $12.63^{\mathrm{a}}$ & $48.49^{a}$ & $5.06^{\mathrm{a}}$ & $14.24^{\mathrm{a}}$ \\
\hline 2 & 1 & $47.19^{\mathrm{a}}$ & $5.66^{\mathrm{ab}}$ & $5.10^{\mathrm{b}}$ & $45.39^{\mathrm{a}}$ & $3.08^{\mathrm{a}}$ & $5.26^{b}$ \\
\hline 2 & 2 & $54.59^{\mathrm{a}}$ & $6.20^{\mathrm{ab}}$ & $5.14^{b}$ & $58.38^{a}$ & $5.36^{\mathrm{a}}$ & $5.49^{b}$ \\
\hline 2 & 3 & $46.91^{\mathrm{a}}$ & $4.93^{b}$ & $5.47^{b}$ & $60.50^{\mathrm{a}}$ & $4.53^{\mathrm{a}}$ & $6.25^{\mathrm{b}}$ \\
\hline 3 & 1 & $56.77^{a}$ & $6.90^{\mathrm{ab}}$ & $15.17^{a}$ & $56.09^{a}$ & $5.03^{\mathrm{a}}$ & $15.75^{\mathrm{a}}$ \\
\hline 3 & 2 & $52.18^{a}$ & $6.48^{\mathrm{ab}}$ & $12.74^{\mathrm{a}}$ & $56.40^{\mathrm{a}}$ & $5.30^{\mathrm{a}}$ & $14.37^{\mathrm{a}}$ \\
\hline 3 & 3 & $52.36^{a}$ & $5.61^{\mathrm{ab}}$ & $14.17^{a}$ & $48.32^{\mathrm{a}}$ & $3.87^{\mathrm{a}}$ & $15.16^{\mathrm{a}}$ \\
\hline $\mathrm{LSD}_{0.05}$ & & 25.16 & 2.09 & 4 & 25.39 & 1.64 & 5.3 \\
\hline \multicolumn{8}{|c|}{ Planting date } \\
\hline October & & $55.13^{a}$ & $8.17^{\mathrm{a}}$ & $10.09^{b}$ & $27.12^{b}$ & $3.58^{b}$ & $6.82^{b}$ \\
\hline November & & $54.36^{a}$ & $4.52^{b}$ & $12.23^{a}$ & $81.71^{\mathrm{a}}$ & $5.94^{\mathrm{a}}$ & $17^{\mathrm{a}}$ \\
\hline $\mathrm{LSD}_{0.05}$ & & 7.27 & 0.87 & 1.68 & 13.37 & 1.27 & 2.28 \\
\hline
\end{tabular}

$L S D_{0.05}$, least significant difference; L1, Veg1; L2, M217; L3, Qukawa; T1, O\% Zn; T2, Zn at 50\%; T3, Zn at $100 \%$.

Means with different superscript letters within the same column are significantly different $(\mathrm{p}<0.05)$. 


\section{Reproductive parameters}

\section{Bien Donne}

The effect of cowpea lines on the measured reproductive parameters is presented in Table 5. A non-significant effect within each cowpea line in response to the different $\mathrm{Zn}$ levels was observed in most measured reproductive parameters. Line 1 at $Z n$ level 1 produced the most pods but these results were not significantly different to the other lines at the same Zn level. Generally, more pods were produced after the first planting date, thereby increasing the pod weight for all treatments at Bien Donne'.

The results of the analysed seed chemical composition of the three cowpea lines are presented in Table 6. No differences were found in the accumulation of potassium among cowpea lines. The three $\mathrm{Zn}$ application levels did not have a significant effect on the seed mineral content within each line. However, a significant decrease in iron content from $88.75 \mathrm{mg} / \mathrm{kg}$ to $77.50 \mathrm{mg} / \mathrm{kg}$ was observed with the application of Zn in Line 1, representing 12.7\% Zn content accumulation. Contradicting results were observed for Line 2 (Table 6). The first planting produced significantly higher seed mineral contents of phosphorus, iron, zinc and calcium.

\section{Nietvoorbi]}

At Nietvoorbij, Line 1 produced the most harvested pods per plant, and thereby the highest pod weight (Table 7). Lines 1 and 3 obtained the highest number of seeds per pod; a similar trend was observed in pod length with Line 2 obtaining significantly fewer seeds per pod and lower pod weight. The second planting produced significantly more seeds per pod, and greater pod length and 100-seed weight at this location.

The three cowpea lines differed significantly in their measured seed mineral contents, except for nitrogen (Table 8). Variation of seed mineral content was observed between the different cowpea lines but the nonsignificant response to $\mathrm{Zn}$ application was observed for most of the minerals measured. However, application of $\mathrm{Zn}$ at Level 3 significantly increased iron in Line 2 compared to application of $\mathrm{Zn}$ at Level 2, with iron concentrations of $97.75 \mathrm{mg} / \mathrm{kg}$ and $83.50 \mathrm{mg} / \mathrm{kg}$, respectively. A significantly lower Zn content was observed when Zn fertiliser (Level 2) was applied to Line 1 . The first planting produced significantly higher phosphorus and sodium contents.

\section{Seed yield}

The average seed yield of the three cowpea lines was $60.7 \mathrm{~kg} / \mathrm{ha}$ and $1184.2 \mathrm{~kg} / \mathrm{ha}$ at Nietvoorbij and Bien Donne', respectively. The average seed yield per hectare (ha) across the locations is presented in Table 9. At both locations, Lines 1 and 3 performed significantly better than Line 2. At Bien Donne', Line 3 showed a significant $46.6 \%$ yield increase compared with Line 2. Remarkably, average seed yield at Bien Donne' was about $58 \%$ more than that at Nietvoorbij. The results of the current study generally indicate a very poor performance of Line 2 at Nietvoorbij with up to $87 \%$ in seed yield compared with that of Line 1 .

Table 5: Influence of zinc application and cowpea line on reproductive parameters at Bien Donne

\begin{tabular}{|c|c|c|c|c|c|c|c|}
\hline Cowpea line & Zinc level & $\begin{array}{l}\text { Number of pods/ } \\
\text { plant }\end{array}$ & $\begin{array}{l}\text { Total harvested } \\
\text { pods }\end{array}$ & $\begin{array}{l}\text { Pod weight } \\
\text { (g) }\end{array}$ & $\begin{array}{l}\text { Number of seeds/ } \\
\text { pod }\end{array}$ & $\begin{array}{l}\text { Pod length } \\
\text { (cm) }\end{array}$ & $\begin{array}{l}\text { 100-seed weight } \\
\text { (g) }\end{array}$ \\
\hline 1 & 1 & $30.02^{\mathrm{a}}$ & $1046.1^{\text {a }}$ & $2086.3^{\mathrm{a}}$ & $16.53^{\mathrm{ab}}$ & $16.35^{b}$ & $11.4^{b}$ \\
\hline 1 & 2 & $34.62^{\mathrm{a}}$ & $1027.0^{a b}$ & $2221.5^{\mathrm{a}}$ & $16.71^{\mathrm{a}}$ & $16.42^{b}$ & $11.5^{\mathrm{b}}$ \\
\hline 1 & 3 & $31.61^{\mathrm{a}}$ & $995.5^{\mathrm{ab}}$ & $2019.3^{\mathrm{a}}$ & $15.75^{\mathrm{abc}}$ & $16.18^{b}$ & $12.2^{b}$ \\
\hline 2 & 1 & $25.99^{a}$ & $831.9^{\mathrm{abc}}$ & $1021.9^{b}$ & $9.26^{d}$ & $11.97^{\circ}$ & $14^{a}$ \\
\hline 2 & 2 & $30.02^{\mathrm{a}}$ & $780.9^{b c}$ & $1104.7^{b}$ & $9.65^{d}$ & $11.78^{c}$ & $13.8^{\mathrm{a}}$ \\
\hline 2 & 3 & $32.72^{\mathrm{a}}$ & $598.0^{c}$ & $973.8^{b}$ & $9.37^{d}$ & $11.52^{c}$ & $13.4^{\mathrm{a}}$ \\
\hline 3 & 1 & $19.64^{\mathrm{a}}$ & $854.3^{\mathrm{ab}}$ & $2157.5^{\mathrm{a}}$ & $15.49^{\mathrm{bc}}$ & $17.81^{\mathrm{a}}$ & $13.7^{\mathrm{a}}$ \\
\hline 3 & 2 & $21.61^{\mathrm{a}}$ & $869.6^{\mathrm{ab}}$ & $2229.7^{\mathrm{a}}$ & $14.92^{\mathrm{c}}$ & $17.56^{\mathrm{a}}$ & $14^{\mathrm{a}}$ \\
\hline 3 & 3 & $20.17^{a}$ & $926.6^{\mathrm{ab}}$ & $2368.4^{\mathrm{a}}$ & $15.54^{\mathrm{bc}}$ & $17.57^{\mathrm{a}}$ & $13.4^{\mathrm{a}}$ \\
\hline $\mathrm{LSD}_{0.05}$ & & 15.26 & 246.59 & 545.43 & 51.08 & 0.57 & 1.08 \\
\hline \multicolumn{8}{|c|}{ Planting date } \\
\hline October & & $28.86^{a}$ & $936.32^{\mathrm{a}}$ & $1926.8^{a}$ & $13.89^{\mathrm{a}}$ & $15.28^{a}$ & $12.6^{\mathrm{b}}$ \\
\hline November & & $25.82^{\mathrm{a}}$ & $835.10^{\circ}$ & $1673.4^{b}$ & $13.50^{\mathrm{a}}$ & $15.27^{\mathrm{a}}$ & $13.49^{a}$ \\
\hline $\mathrm{LSD}_{0.05}$ & & 4.63 & 73.82 & 209.74 & 0.6 & 0.33 & 0.52 \\
\hline
\end{tabular}

$L S D_{0.05}$ least significant difference; $L 1$, Veg1; L2, M217; L3, Qukawa; T1, 0\% Zn; T2, Zn at 50\%; T3, Zn at $100 \%$.

Means with different superscript letters within the same column are significantly different $(p<0.05)$. 
Table 6: Cowpea seed mineral content after zinc application at Bien Donne'

\begin{tabular}{|c|c|c|c|c|c|c|c|c|c|}
\hline \multirow{2}{*}{ Cowpea line } & \multirow{2}{*}{ Zinc level } & \multicolumn{3}{|c|}{$\%$} & \multicolumn{5}{|c|}{$\mathrm{mg} / \mathrm{kg}$} \\
\hline & & N & $\mathbf{P}$ & K & $\mathrm{Na}$ & $\mathrm{Fe}$ & $\mathrm{Zn}$ & $\mathrm{Ca}$ & $\mathrm{Mg}$ \\
\hline 1 & 1 & $3.96^{a b}$ & $0.5^{\text {cd }}$ & $1.57^{\mathrm{a}}$ & $119.25^{\mathrm{ab}}$ & $88.75^{\mathrm{a}}$ & $35.00^{b}$ & $0.12^{\mathrm{bc}}$ & $0.21^{\mathrm{a}}$ \\
\hline 1 & 2 & $4.08^{a}$ & $0.49^{d}$ & $1.53^{\mathrm{a}}$ & $126.00^{\mathrm{a}}$ & $84.25^{\mathrm{ab}}$ & $39.00^{\mathrm{ab}}$ & $0.11^{\mathrm{cd}}$ & - \\
\hline 1 & 3 & $3.96^{a b}$ & - & $1.46^{\mathrm{a}}$ & $128.50^{\mathrm{a}}$ & $77.50^{\mathrm{bc}}$ & $37.25^{b}$ & $0.10^{\mathrm{cd}}$ & $0.20^{\mathrm{b}}$ \\
\hline 2 & 1 & $3.86^{a b}$ & $0.52^{\mathrm{bcd}}$ & $1.47^{\mathrm{a}}$ & $107.25^{\mathrm{ab}}$ & $67.00^{c}$ & $38.25^{\mathrm{ab}}$ & $0.15^{\mathrm{a}}$ & $0.18^{c}$ \\
\hline 2 & 2 & $3.78^{b}$ & $0.53^{\mathrm{abc}}$ & $1.48^{\mathrm{a}}$ & $106.25^{\mathrm{ab}}$ & $86.50^{\mathrm{ab}}$ & $36.25^{b}$ & $0.14^{\mathrm{ab}}$ & $0.20^{\mathrm{b}}$ \\
\hline 2 & 3 & $3.88^{\mathrm{ab}}$ & $0.53^{\mathrm{ab}}$ & $1.49^{\mathrm{a}}$ & $77.75^{b}$ & $82.25^{\mathrm{ab}}$ & $28.75^{\mathrm{ab}}$ & $0.15^{\mathrm{a}}$ & $0.19^{b c}$ \\
\hline 3 & 1 & $3.94^{\mathrm{ab}}$ & $0.54^{\mathrm{ab}}$ & $1.52^{\mathrm{a}}$ & $111.25^{\mathrm{ab}}$ & $79.25^{\mathrm{ab}}$ & $39.00^{\mathrm{ab}}$ & $0.10^{d}$ & $0.20^{\mathrm{b}}$ \\
\hline 3 & 2 & $4.08^{a}$ & $0.56^{a}$ & $1.57^{\mathrm{a}}$ & $107.25^{\mathrm{ab}}$ & $85.00^{\mathrm{ab}}$ & $42.00^{\mathrm{a}}$ & $0.11^{\mathrm{cd}}$ & $0.20^{\mathrm{b}}$ \\
\hline 3 & 3 & $3.98^{\mathrm{ab}}$ & $0.53^{\mathrm{abc}}$ & $1.53^{\mathrm{a}}$ & $80.75^{b}$ & $80.50^{\mathrm{ab}}$ & $38.25^{a b}$ & $0.11^{\mathrm{cd}}$ & $0.20^{\mathrm{b}}$ \\
\hline $\mathrm{LSD}_{0.05}$ & & 0.26 & 0.03 & 0.13 & 37.56 & 10.85 & 4.12 & 0.02 & 0.01 \\
\hline \multicolumn{10}{|c|}{ Planting date } \\
\hline October & & $3.92^{\mathrm{a}}$ & $0.53^{\mathrm{a}}$ & $1.52^{\mathrm{a}}$ & $118.11^{a}$ & $88.06^{a}$ & $39.56^{\mathrm{a}}$ & $0.13^{\mathrm{a}}$ & $0.19^{\mathrm{a}}$ \\
\hline November & & $3.98^{\mathrm{a}}$ & $0.51^{b}$ & $1.50^{\mathrm{a}}$ & $96.17^{\mathrm{a}}$ & $74.39^{b}$ & $36.83^{b}$ & $0.12^{b}$ & $0.19^{\mathrm{a}}$ \\
\hline $\mathrm{LSD}_{0.05}$ & & 0.15 & 0.02 & 0.04 & 28.19 & 7.35 & 2.28 & 0.01 & 0.01 \\
\hline
\end{tabular}

$L S D_{0.05}$, least significant difference; L1, Veg1; L2, M217; L3, Qukawa; T1, O\% Zn; T2, Zn at 50\%; T3, Zn at 100\%.

Means with different superscript letters within the same column are significantly different $(\mathrm{p}<0.05)$.

Table 7: Influence of zinc application and cowpea line on reproductive parameters at Nietvoorbij

\begin{tabular}{|c|c|c|c|c|c|c|c|}
\hline Cowpea line & Zinc level & $\begin{array}{c}\text { Number of pods/ } \\
\text { plant }\end{array}$ & $\begin{array}{l}\text { Total harvested } \\
\text { pods }\end{array}$ & $\begin{array}{l}\text { Pod weight } \\
\text { (g) }\end{array}$ & $\begin{array}{c}\text { Number of seeds/ } \\
\text { pod }\end{array}$ & $\begin{array}{l}\text { Pod length } \\
\text { (cm) }\end{array}$ & $\begin{array}{l}\text { 100-seed weight } \\
\text { (g) }\end{array}$ \\
\hline 1 & 1 & $38.31^{a}$ & $542.20^{\mathrm{ab}}$ & $1074.2^{\mathrm{ab}}$ & $13.78^{\mathrm{a}}$ & $16.14^{a}$ & $12.22^{\mathrm{abcd}}$ \\
\hline 1 & 2 & $25.47^{\mathrm{abc}}$ & $640.57^{\mathrm{a}}$ & $1372.5^{\mathrm{a}}$ & $13.27^{\mathrm{a}}$ & $15.78^{a}$ & $12.11^{\mathrm{abcd}}$ \\
\hline 1 & 3 & $33.15^{\mathrm{ab}}$ & $647^{a}$ & $1296.7^{\mathrm{a}}$ & $13.95^{\mathrm{a}}$ & $16.02^{\mathrm{a}}$ & $12.5^{\mathrm{abc}}$ \\
\hline 2 & 1 & $12.01^{d}$ & $121.50^{\mathrm{d}}$ & $121.4^{c}$ & $5.23^{c}$ & $8.24^{c}$ & $9.1^{d}$ \\
\hline 2 & 2 & $17.27^{\mathrm{cd}}$ & $121.67^{\mathrm{d}}$ & $127.3^{c}$ & $7.87^{\mathrm{b}}$ & $11.58^{b}$ & $10.6^{b d c}$ \\
\hline 2 & 3 & $12.29^{d}$ & $180.38^{c d}$ & $180.2^{c}$ & $6.25^{b c}$ & $9.26^{c}$ & $9.4^{c d}$ \\
\hline 3 & 1 & $17.25^{\mathrm{cd}}$ & $417.50^{\mathrm{b}}$ & $1006.2^{\mathrm{ab}}$ & $12.74^{\mathrm{a}}$ & $17.23^{\mathrm{a}}$ & $14.1^{\mathrm{a}}$ \\
\hline 3 & 2 & $21.13^{\text {bcd }}$ & $431.80^{\mathrm{b}}$ & $1029.5^{\mathrm{ab}}$ & $13.65^{\mathrm{a}}$ & $17.79^{\mathrm{a}}$ & $14.22^{\mathrm{a}}$ \\
\hline 3 & 3 & $14.39^{\mathrm{cd}}$ & $356.20^{\mathrm{bc}}$ & $838.1^{b}$ & $12.24^{a}$ & $16.87^{a}$ & $12.9^{a b}$ \\
\hline $\mathrm{LSD}_{0.05}$ & & 12.85 & 195.61 & 421.79 & 1.75 & 2.07 & 3.3 \\
\hline \multicolumn{8}{|c|}{ Planting date } \\
\hline October & & $27.61^{a}$ & $405.64^{a}$ & $854.90^{\mathrm{a}}$ & $10.56^{b}$ & $13.71^{b}$ & $10.74^{b}$ \\
\hline November & & $15.52^{b}$ & $374.91^{\mathrm{a}}$ & $763.16^{\mathrm{a}}$ & $11.75^{\mathrm{a}}$ & $15.09^{a}$ & $12.98^{\mathrm{a}}$ \\
\hline $\mathrm{LSD}_{0.05}$ & & 5.55 & 63.67 & 179.37 & 0.83 & 0.9 & 1.37 \\
\hline
\end{tabular}

$L S D_{0.05^{\prime}}$ least significant difference; L1, Veg1; L2, M217; L3, Qukawa; T1, O\% Zn; T2, Zn at 50\%; T3, Zn at $100 \%$.

Means with different superscript letters within the same column are significantly different $(\mathrm{p}<0.05)$. 
Table 8: $\quad$ Cowpea seed mineral content after zinc application at Nietvoorbij

\begin{tabular}{|c|c|c|c|c|c|c|c|c|c|}
\hline \multirow{2}{*}{$\begin{array}{l}\text { Cowpea } \\
\text { line }\end{array}$} & \multirow{2}{*}{$\begin{array}{l}\text { Zinc } \\
\text { level }\end{array}$} & \multicolumn{3}{|c|}{$\%$} & \multicolumn{5}{|c|}{$\mathrm{mg} / \mathrm{kg}$} \\
\hline & & $\mathbf{N}$ & $\mathbf{P}$ & K & $\mathrm{Na}$ & $\mathrm{Fe}$ & $\mathrm{Zn}$ & $\mathrm{Ca}$ & $\mathrm{Mg}$ \\
\hline 1 & 1 & $3.77^{a}$ & $0.47^{b}$ & $1.55^{\mathrm{ab}}$ & $321.5^{\mathrm{abc}}$ & $75.50^{c}$ & $62.00^{\mathrm{a}}$ & $0.14^{c}$ & - \\
\hline 1 & 2 & $3.77^{\mathrm{a}}$ & $0.51^{\mathrm{ab}}$ & $1.59^{\mathrm{a}}$ & $356.75^{\mathrm{a}}$ & $75.50^{c}$ & $57.50^{b}$ & - & $0.20^{\mathrm{a}}$ \\
\hline 1 & 3 & $3.88^{\mathrm{a}}$ & $0.49^{\mathrm{ab}}$ & $1.59^{\mathrm{a}}$ & $346.75^{\mathrm{ab}}$ & $73.00^{c}$ & $54.50^{\mathrm{bc}}$ & $0.13^{c}$ & $0.20^{\mathrm{a}}$ \\
\hline 2 & 1 & $3.81^{\mathrm{a}}$ & $0.51^{\mathrm{ab}}$ & $1.53^{\mathrm{abc}}$ & $277.50^{\mathrm{abcd}}$ & $92.00^{\mathrm{ab}}$ & $50.00^{\text {de }}$ & $0.17^{\mathrm{ab}}$ & $0.18^{b}$ \\
\hline 2 & 2 & $3.86^{\mathrm{a}}$ & $0.52^{\mathrm{a}}$ & $1.55^{\mathrm{ab}}$ & $287.50^{\mathrm{abc}}$ & $83.50^{b c}$ & $49.25^{\mathrm{e}}$ & $0.19^{a}$ & $0.19^{b}$ \\
\hline 2 & 3 & $3.79^{a}$ & $0.55^{\mathrm{a}}$ & $1.54^{\mathrm{ab}}$ & $285.25^{\mathrm{abc}}$ & $97.75^{\mathrm{a}}$ & $51.25^{\text {cde }}$ & $0.18^{a}$ & $0.19^{b}$ \\
\hline 3 & 1 & $3.94^{\mathrm{a}}$ & $0.48^{b}$ & $1.45^{c}$ & $210.25^{\text {cd }}$ & $77.00^{c}$ & $53.25^{\mathrm{cd}}$ & $0.14^{c}$ & $0.19^{b}$ \\
\hline 3 & 2 & $3.97^{\mathrm{a}}$ & $0.49^{\mathrm{ab}}$ & $1.45^{c}$ & $159.00^{d}$ & $76.00^{c}$ & $54.00^{\mathrm{bc}}$ & $0.13^{c}$ & $0.18^{b}$ \\
\hline 3 & 3 & $3.82^{\mathrm{a}}$ & $0.51^{\mathrm{ab}}$ & $1.48^{\mathrm{bc}}$ & $229.00^{\mathrm{bcd}}$ & $77.50^{c}$ & $54.25^{\mathrm{bc}}$ & $0.15^{b c}$ & $0.19^{b}$ \\
\hline $\mathrm{LSD}_{0.05}$ & & 0.39 & 0.07 & 0.09 & 120.77 & 13.45 & 3.76 & 0.03 & 0.01 \\
\hline \multicolumn{10}{|c|}{ Planting date } \\
\hline October & & $3.85^{\mathrm{a}}$ & $0.52^{\mathrm{a}}$ & $1.54^{\mathrm{a}}$ & $286.56^{a}$ & $80.67^{a}$ & $53^{a}$ & $0.15^{\mathrm{a}}$ & $0.19^{\mathrm{a}}$ \\
\hline November & & $3.84^{\mathrm{a}}$ & $0.50^{\mathrm{b}}$ & $1.51^{\mathrm{a}}$ & $263.11^{\mathrm{b}}$ & $81.06^{a}$ & $53.53^{\mathrm{a}}$ & $0.15^{\mathrm{a}}$ & $0.19^{\mathrm{a}}$ \\
\hline $\mathrm{LSD}_{0.05}$ & & 0.2 & 0.01 & 0.05 & 13.89 & 7.49 & 2.8 & 0.01 & 0.003 \\
\hline
\end{tabular}

$L S D_{0.05}$, least significant difference; $L 1$, Veg1; L2, M217; L3, Qukawa; T1, $0 \%$ Zn; T2, Zn at 50\%; T3, Zn at 100\%.

Means with different superscript letters within the same column are significantly different $(p<0.05)$.

Table 9: $\quad$ Average seed yield ( $\mathrm{kg} / \mathrm{ha})$ across the two locations

\begin{tabular}{|c|c|c|c|}
\hline \multirow{2}{*}{$\begin{array}{c}\text { Cowpea } \\
\text { line }\end{array}$} & \multirow{2}{*}{$\begin{array}{c}\mathrm{Zn} \\
\text { level }\end{array}$} & Bien Donne' & \multirow{2}{*}{$\begin{array}{l}\text { Nietvoorbij } \\
\text { Yield (kg/ha) }\end{array}$} \\
\hline & & Yield (kg/ha) & \\
\hline 1 & 1 & $1043.15^{\mathrm{a}}$ & $537.1^{\mathrm{ab}}$ \\
\hline 1 & 2 & $1110.75^{\mathrm{a}}$ & $686.25^{\mathrm{a}}$ \\
\hline 1 & 3 & $1009.65^{\mathrm{a}}$ & $648.35^{a}$ \\
\hline 2 & 1 & $510.95^{b}$ & $60.7^{c}$ \\
\hline 2 & 2 & $552.35^{b}$ & $63.65^{c}$ \\
\hline 2 & 3 & $486.9^{b}$ & $90.1^{c}$ \\
\hline 3 & 1 & $1078.75^{\mathrm{a}}$ & $503.1^{\mathrm{ab}}$ \\
\hline 3 & 2 & $1114.85^{\mathrm{a}}$ & $514.75^{\mathrm{ab}}$ \\
\hline 3 & 3 & $1184.2^{\mathrm{a}}$ & $419.05^{b}$ \\
\hline $\mathrm{LSD}_{0.05}$ & & 272.72 & 210.90 \\
\hline \multicolumn{4}{|c|}{ Planting date } \\
\hline October & & $963.4^{\mathrm{a}}$ & $427.45^{a}$ \\
\hline November & & $836.7^{b}$ & $381.58^{a}$ \\
\hline $\mathrm{LSD}_{0.05}$ & & 104.87 & 89.69 \\
\hline
\end{tabular}

$L S D_{0.05}$, least significant difference; $L 1$, Veg1; $L 2, M 217 ; \quad L 3$, Qukawa; $T 1,0 \% Z n ; T 2$, Zn at $50 \% ; T 3$, Zn at $100 \%$.

Means with different superscript letters within the same column are significantly different $(p<0.05)$.

\section{Discussion}

The qualitative and quantitative traits of the cowpea lines studied varied (Table 1); the traits outline the morphology of the three cowpea lines studied. Morphological traits of cowpea lines and the importance of these traits are well documented by Egbadzor et al. ${ }^{29}$ Veg1 and Qukawa had the highest mean germination rate, while M217 had the lowest number of germinated plants per experimental unit. The obtained results were similar to those reported by Wada and Abubakar ${ }^{30}$ who did a germination test on different cowpea lines and concluded that seed size and viability of seed are the factors that affect germination on different cowpea lines. The results of the study indicate that Veg1 and Qukawa can successfully be sown early (2 October) or late (2 November) in the Western Cape region (Figure 1). Furthermore, M217 will successfully germinate to its highest capacity if sown later (2 November) in the growing season when temperatures are between $10^{\circ} \mathrm{C}$ and $30^{\circ} \mathrm{C}$. Germination rate was significantly affected by soil type; in sandy loam soil germination was more efficient than in sandy clay loam soils as a result of the different soil textural percentages at the two locations. Similar results were obtained from a study done by Pahla et al. ${ }^{27}$ who found a higher percentage of germination and emergence on sandy loam soils.

Vegetative parameters of the three cowpea lines differed significantly, which could be associated with genotypic make-up, season or location. ${ }^{31,32}$ There were no significant differences in the number of leaves per plant observed at Bien Donne' or at Nietvoorbij. Comparable findings on the number of leaves were documented by Olatunji et al. ${ }^{33}$ The two farms had soils of different textures. We have shown that plants at Nietvoorbij, which has clay loam soils, had the most branches and greatest plant heights compared with those of Bien Donne'. The results regarding the number of branches are in agreement with those of Shiringani ${ }^{24}$.

Veg1 and Qukawa showed the greatest plant heights and M217 was shorter across the two locations. This difference could be associated with the genetic make-up of each line, as the morphological trait of the two lines showed a similar growth pattern. According to Egbadzor et al. ${ }^{29}$, plants that are classified as indeterminate are most vigorous. Veg1 had an indeterminate or spreading type plant pattern which concurs with the above statement of Egbadzor et al. According to our results, zinc 
fertiliser application at the onset of flowering did not have a significant effect on the vegetative growth of cowpea. These findings are contrary to the results of Elowad and Hall ${ }^{4}$, who concluded that early flowering soil application of fertiliser increases the number of branches and pods of cowpea lines. Veg1 and Qukawa were recognised as the best performing lines for cultivation at both locations. Parameters such as the number of pods per plant and number of seeds per pod contributed to the total yield harvested of the two lines. The mean number of pods was in the range of 14-33 per plant. Similar results on 12 genotypes were reported by Peksen and Peksen ${ }^{34}$. Generally, Veg1 was the best performing line across all cowpea lines and M217 was the worst. Shiringani ${ }^{24}$ obtained contrary results on the performance of M217 which had the most pods per plant in two locations in that study. At Nietvoorbij, Veg1 had the most pods per plant and no difference was observed between zinc application levels. This insignificant response to zinc application was observed in most of the parameters measured. The inconsistency in response of all lines to zinc application could be associated with the time of application and the ability to absorb and translocate the nutrients to the sink. ${ }^{35}$ Rathore et al. ${ }^{36}$ stated that the ability of a plant to absorb available zinc in the soil is also influenced by other plant nutrients, which can promote or hinder the plant's ability to absorb and translocate zinc to all parts of the plants. A fascinating finding from our study was that M217 at Bien Donne' obtained the highest 100-seed weight (14 g/100 seed). These weights were significantly higher than those of Veg1 and M217 is regarded as the best performing line. The findings clearly indicate that M217 had the bigger seed size, although it lacked in most of the parameters measured. Bigger seed size is mostly preferred for home consumption. ${ }^{37}$ Aliyu and Makinde ${ }^{38}$ concluded that seed size was related to the number of days to flowering and pod formation period. These findings are in agreement with our observations in the current study, as line M217 was the first line to flower and bear pods. Zinc fertiliser did not have a significant effect on most of the measured parameters, which could be associated with the time of application and the ability of the plant to absorb the fertiliser.

\section{Conclusion}

We evaluated the vegetative and reproductive parameters of three cowpea lines in response to three zinc fertiliser application levels in the Western Cape Boland region. Our results show that Veg1 and Qukawa performed better in the measured parameters than M217. Planting cowpea in November rather than October significantly increased the crops' ability to germinate efficiently, and thereby increased production efficiency. Bien Donne was the best location for the production of cowpea as the total number of harvested seeds was significantly higher for all cowpea lines at Bien Donne' than at Nietvoorbij.

\section{Acknowledgements}

The support and technical guidance of Prof. Lewu and Dr Mulidzi are highly appreciated. The study was supported financially by the Cape Peninsula University of Technology Research Fund, the Agricultural Research Council and the National Research Foundation of South Africa.

\section{Authors' contributions}

N.M., R.A.M. and F.B.L. conceived and planned the experiments. N.M. carried out the experiments. F.B.L., N.M. and R.A.M. contributed to the interpretation of the results. N.M. took the lead in writing the manuscript. All authors provided critical feedback and helped shape the research, analysis and writing.

\section{References}

1. Iqbal A, Khalil IA, Ateeq N, Khan MS. Nutritional quality of important food legumes. Food Chem. 2006;97:331-335. https://doi.org/10.1016/j. foodchem.2005.05.011

2. Sebetha ET, Ayodele VI, Kutu FR, Mariga IK. Yield and protein content of two cowpea varieties grown under different production practices in Limpopo Province, South Africa. Afr J Biotechnol. 2010;9(5):628-634. http://dx.doi. org/10.5897/AJB09.1132

3. Singh BB, Ajeigbe HA, Tarawal SA, Fernandez-Rivera S, Abubakar M. Improving the production and utilization of cowpea as food and fodder. Field Crop Res. 2003;84:169-177. https://doi.org/10.1016/S0378-4290(03)00148-5
4. Elowad HOA, Hall AE. Influence of early and late nitrogen fertilization on yield and nitrogen fixation of cowpea under well-watered and dry field conditions. Field Crop Res. 1987;15:229-244. https://doi.org/10.1016/0378-4290(87)90012-8

5. West DW, Francois LE. Effects of salinity of germination, growth and yield of cowpea. Irrigation Sci. 1982;3:169-175. https://doi.org/10.1007/ BF00446005

6. Ndema NE, Etame J, Taffouo VD, Bilong P. Effects of some physical and chemical characteristics of soil on productivity and yield of cowpea (Vigna unguiculata L. Walp.) in coastal region (Cameroon). Afr J Environ Sci Tech. 2010;4(3):108-118. https://doi.org/10.5897/AJEST09.160

7. Gomez C. Cowpea: Post-harvest operations. In: Mejía D, editor. Post-harvest compendium. Rome: FAO; 2004. Available from: http://www.fao.org/3/aau994e.pdf

8. Mamiro PS, Mbwaga AM, Mamiro DP, Mwanri AW, Kinabo JL. Nutritional quality and utilization of local and improved cowpea varieties in some regions in Tanzania. Afr J Food Agric Nutr Develop. 2011;11(1):4490-4506. https:// doi.org/10.4314/ajfand.v11i1.65876

9. Khalid II, Elhadallou SB, Elkhalifa EA. Composition and functional properties of cowpea (Vigna anguiculata L. Walp) flour and protein isolates. Am J Food Technol. 2012;7(3):113-122. https://doi.org/10.3923/ajtt.2012.113.122

10. Kebe K, Sembene M. Cowpea (Vigna unguiculata (L.) Walp) field infestation by the bruchids (Coleoptera: Bruchidae) in the northern Senegal: Preliminary biological and ecological data. J Appl Biosci. 2011;41:2788-2796. http:// www.m.elewa.org/JABS/2011/41/6.pdf

11. Magloire N. The genetic, morphology and physical evaluation of African cowpea genotypes [MSc thesis]. Bloemfontein: University of the Free State; 2005.

12. Singh AK, Bhatt BP, Sundaram PK, Kumar S, Bahrati RC, Chandra N, et al. Study of site specific nutrients management of cowpea seed production and their effect on soil nutrient status. J Agric Sci. 2012;4(10):191-198. http:// dx.doi.org/10.5539/jas.v4n10p19

13. Moswatsi MS, Kutu FR, Mafeo TP. Response of cowpea to variable rates and methods of zinc application under different field conditions. Afr Crop Sci. 2013;11:757-762.

14. Belane AK, Dakora FD. Elevated concentrations of dietarily-important trace elements and micronutrients in edible leaves and grain of 27 cowpea (Vigna unguilata L. Walp.) genotypes: Implications of human nutrition and health Food Nutr Sci. 2012;3:377-386. http://dx.doi.org/10.4236/fns.2012.33054

15. South African Department of Agriculture, Forestry and Fisheries (DAFF). Production guidelines for cowpea. c2011 [2015 Feb 13]. Available from: http://www.arc.agric.za/arc-gci/Fact\%20Sheets\%20Library/Cowpea\%20 \%20Production\%20guidelines\%20for\%20cowpea.pdf

16. Frankenberger WT, Abdelmagid HM. Kinetic parameters of nitrogen mineralization rates of leguminous crops incorporated into soil. Plant Soil. 1985;87(2):257-271. https://doi.org/10.1007/BF02181865

17. Bloem JF, Trystman G, Smith HJ. Biological nitrogen fixation in resourcepoor agriculture in South Africa. Symbiosis. 2009;48:18-24. https://doi. org/10.1007/BF03179981

18. Chivenge P, Mabhuadhi T, Modi AT, Mafongoya P. The potential role of neglected and underutilised crop species as future crops under water scarce conditions in sub-Sahara Africa. Int J Environ Res Public Health. 2015;12:5685-5711. https://doi.org/10.3390/ijerph120605685

19. Degri MM, Sharah HA, Duada Z. Effects of intercropping pattern and planting date on the performance of two cowpea variety in Dalwa, Maiduguri, Nigeria. Glob J Biosci Biotechnol. 2013;2(4):480-484.

20. Shiringani RP, Shimelis HA. Yield response and stability among cowpea genotype at .three planting dates and test environments. Afr J Agric Res. 2011;6(14):3259-3263.

21. Asiwe JAN. Needs assessment of cowpea production practices, constraints and utilization in South Africa. Afr J Biotechnol. 2009;8(20):5383-5388. https://doi.org/10.5897/AJB09.1293

22. Mabhudhi T, Modi AT. Sowing the seed of knowledge on underutilised crop. The Water Wheel. 2016;15(2):40-41. https://doi.org/10.3390/su9091569

23. Quass CF. Guidelines for the production of cowpeas. Pretoria: Nationa Department of Agriculture; 1995. p. 1-17. 
24. Shiringani RP. Effect of planting date and location on phenology yield and yield components among selected cowpea varieties [MSc thesis]. Tzaneen: University of Limpopo; 2007.

25. International Board for PlantGenetics Resources (IBPGR). Cowpea descriptors. Rome: IBPGR; 1983. Available from: http://www.bioversityinternational.org/ uploads/tx_news/Descriptors_for_cowpea_377.pdf

26. Fourie JC. Soil management in the Breede River Valley wine grape region, South Africa. Grapevine performance. S Afr J Enol Vitic. 2011;33(1):60-70. http://dx.doi.org/10.21548/32-1-1367

27. Pahla I, Muziri T, Chinyise T, Muzemu S, Chitambu J. Effect of soil type and different pre-sowing treatments on seedling and vigour of Acacia seiberana. Int J Plant Res. 2014;4(2):51-55. http://dx.doi.org/10.5923/j. plant.20140402.02

28. SAS Institute. The statistical procedure manual. Cary, NC: SAS; 2012. Available from: https://support.sas.com/documentation/onlinedoc/stat/121/ intro.pdf

29. Egbadzor KF, Danquah EY, Ofori K, Yeboah M, Offei SK. Diversity in 118 cowpea [Vigna unguiculata (L.) Walp] accessions assessed with 16 morphological traits. Int J Plant Breed Genet. 2014;8(1):13-24. https://doi. org/10.3923/ijpbg.2014.13.24

30. Wada BY, Abubakar BY. Germination studies in varieties of Vigna unguiculata L. Walp. (cowpea) of northern Nigeria. Pak J Biol Sci. 2013;16(20):12201222. https://doi.org/10.3923/pjbs.2013.1220.1222

31. Ayan I, Mut H, Basaran U, Acar Z, Asci 00. Forage potential of cowpea (Vigna unguiculata L. Walp). Turk J Field Crops. 2012;17(2):135-138. http:// dergipark.gov.tr/download/article-file/158677
32. Gerreno AS, Adebola PO, Van Renseburg WSJ, Laurie SM. Genetic variability in cowpea (Vigna unguiculata (L.) Walp) genotypes. S Afr J Plant Soil. 2015;32(2):164-174. https://doi.org/10.1080/02571862.2015.1014435

33. Olatunji OA, Okunlola GO, Komolafe ET, Afalabi AW, Tariq A, Odeleye AA. Yield and growth characteristics of cowpea (Vigna unguiculata) as affected by prior heat stress and nutrient addition. Afr J Agric Res. 2016;11(43):4269-4276. https://doi.org/10.5897/AJAR2016.11651

34. Peksen E, Peksen A. Evaluation of vegetable cowpea (Vigna unguiculata (L.) Walp.) Breeding lines for cultivar development. Igdir Univ J Inst Sci Tech. 2012;2(4):9-18. http://www.igdir.edu.tr/Addons/Resmi/announc/237/9-18.pdf

35. Naim AME, Jabereldar AA, Ahmed SE, Ismaeil FM, Ibrahim EA. Determination of suitable variety and plants per stand of cowpea (Vigna unguiculata I. Walp) in the sandy soils, Sudan. Adv Life Sci. 2012;2(1):1-5. https://doi. org/10.5923/j.als.20120201.01

36. Rathore DK, Kumar R, Singh M, Meena VK, Kumar U, Gupta PS, et al. Phosphorus and zinc fertilization I fodder cowpea - A review. Agric Rev. 2015;36(4):333-338. https://doi.org/10.18805/ag.v36i4.6670

37. Doumbia IZ, Akromah R, Asibuo JY. Comparative study of cowpea germplasms diversity from Ghana and Mali using morphological characteristic. J Plant Breed Genet. 2013;1(3):139-147. http://article.sapub. org/10.5923.j.jjaf.20140402.12.html

38. Aliyu OM, Makinde BO. Phenotypic analysis of seed yield and yield components in cowpea (Vigna unguiculata L., Walp). Plant Breed Biotech. 2016;4(2):252-261. https://doi.org/10.9787/PBB.2016.4.2.252 